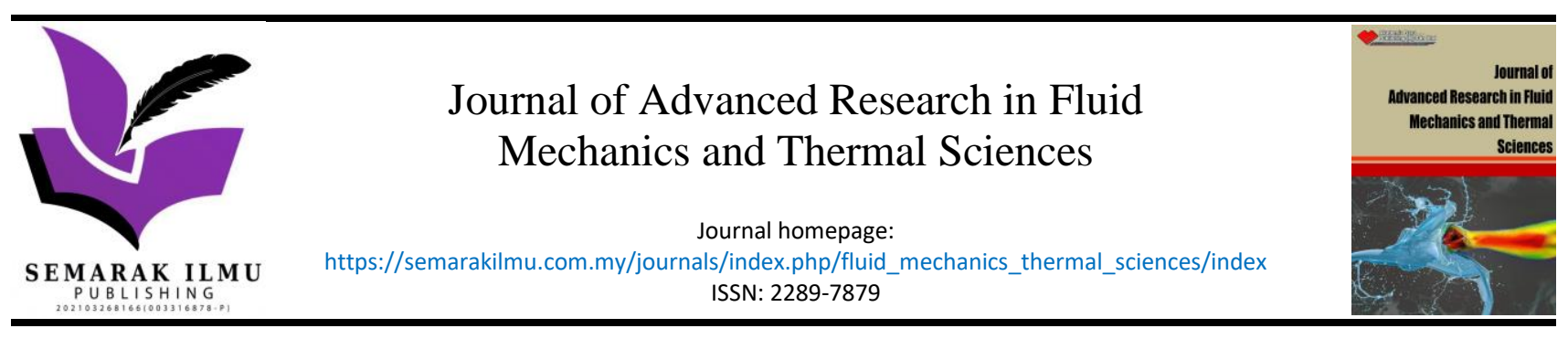

\title{
A Review on Generation and Mitigation of Airfoil Self-Induced Noise
}

\author{
Mohamed Ibren ${ }^{1}$, Amelda Dianne Andan ${ }^{1, *}$, Waqar Asrar $^{1}$, Erwin Sulaeman ${ }^{1}$ \\ 1 Department of Mechanical Engineering, Faculty of Engineering, International Islamic University Malaysia, 53100, Kuala Lumpur, Malaysia
}

\section{ARTICLE INFO}

Article history:

Received 14 August 2021

Received in revised form 10 November 2021

Accepted 15 November 2021

Available online 21 December 2021

Keywords:

Airfoil self-noise; aeroacoustics; porous

\section{ABSTRACT}

\begin{abstract}
A review on passive acoustic control of airfoil self-noise by means of porous trailing edge is presented. Porous surfaces are defined using various terms such as porosity, permeability, resistivity, porosity constant, dimensionless permeability, flow control severity and tortuosity. The primary purpose of this review paper is to provide key findings regarding the sources and mitigation techniques of self-induced noise generated by airfoils. In addition, various parametric design concepts were presented, which are critically important for porous-airfoil design specifications. Most research focus on experimentation with some recent efforts on numerical simulations. Detail study on flow topology is required to fully understand the unsteady flow nature. In general, noise on the airfoil surface is linked to the vortex shedding, instabilities on the surface, as well as feedback mechanism. In addition, acoustic scattering can be minimized by reducing extent of the porous region from the trailing edge while increasing resistivity. Moreover, blowing might also be another means of reducing noise near the trailing edge. Ultimately, understanding the flow physics well provides a way to unveil the unknowns in self-induced airfoil noise generation, mitigation, and control.
\end{abstract}

\section{Introduction}

The aviation sector has been continuously improving and reducing noise associated with aircraft wings and helicopter blades. These efforts have been deliberated carefully by acoustically tailored materials and/or altering the design configurations. Porosity, serration, morphing, shape optimization, and finlets are amongst the techniques used to mitigate the noise generated at the trailing edge of the airfoils. In addition, several features such as fringes and downy wing surfaces have shown significant noise reduction within audible range of their prey and themselves. However, detail physical analysis is needed for better understanding [1]. Computer simulation is one of the methods used to understand the physics of the flow structure over airfoils since it is cost-efficient. Thus, the method can investigate better ways of mitigating the effects of the noise. Moreover, experimentation came in handy to validate the simulation data and visualize the actual scenario. Noise is unpleasant and causes discomfort to passengers as well as the residential areas. Reduction and control of noise are efforts to achieve sustainable transportation, which has a low impact on

\footnotetext{
* Corresponding author.

E-mail address: ameldadianne@iium.edu.my
}

https://doi.org/10.37934/arfmts.90.1.163178 
users and the environment. Quiet take-off and landing for the commercial airplanes near the population are one of the main goals of the International Civil Aviation Organization (ICAO). Therefore, ICAO is planning to reduce the number of individuals who are affected by aircraft noise.

The characteristics and behavior of tonal emissions at low Reynolds number ( $R e \approx 10^{4}$ ) differ from that at higher Reynolds number [2]. Flow separation and laminar separation bubble (LSB) has tremendous effects on the flow topology over airfoils operating at lower Reynolds number. At lower Reynolds number, the separated laminar boundary layer (LBL) rapidly transits to turbulence by forming an unsteady vortex structure and is usually followed by flow reattachment [3]. Thus, creating laminar separation bubbles. In addition, the laminar separation bubble is dependent not only on the Reynolds number but also on the angle of attack [4]. The size and location of the separation bubble vary as the Reynolds number and angle of attack increase, hence changing the fluid flow structure over a body [5]. Laminar separation bubbles can form on both side of the airfoil at small angles of attack.

Boundary layer separation gradually decreases as the angle of attack increases, which leads to a decrease in the size and extension of the laminar bubble separation on the airfoil pressure side (Figure 1). Also, increasing the angle of attack, laminar separation points rapidly shifted from the trailing edge to the mid chord and eventually to the leading edge on the airfoil suction side [6]. These cause flow transition to move upstream on the suction side whereas, the laminar boundary layer on the pressure side extends close to the trailing edge before turning into a laminar separation bubble [7]. Moreover, the size of the laminar separation bubble is also affected by airfoil profile and thickness; varying the angle of attack was found to significantly change the flow structure and the aerodynamic performance [8].

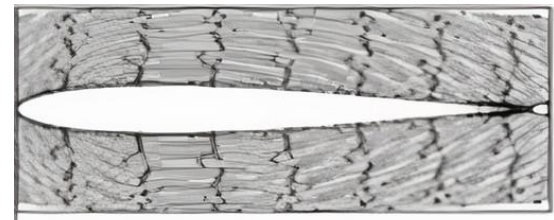

(๑) $a \div 0^{\circ}$

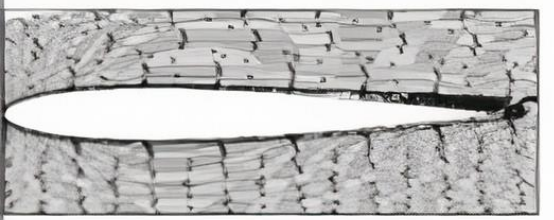

(c) $a \infty 1.5^{\circ}$

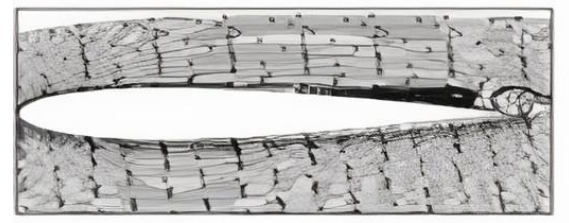

(c) $a .2 .5^{\circ}$

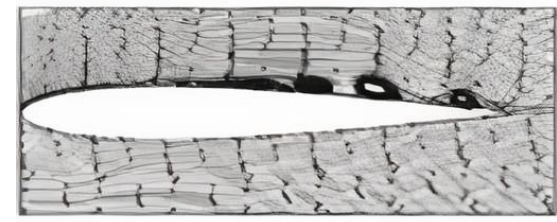

(8) a $07.0^{\circ}$

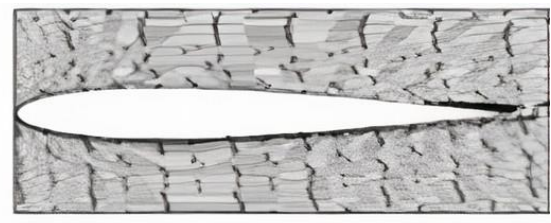

(b) $\propto 0.5^{\circ}$

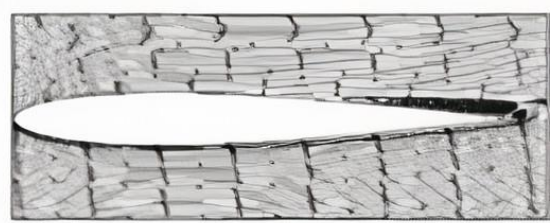

(d) $a=2.0^{\circ}$

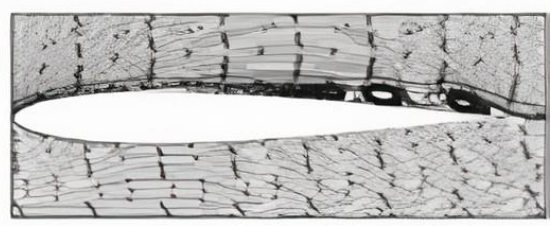

( $) \propto=3.0^{\circ}$

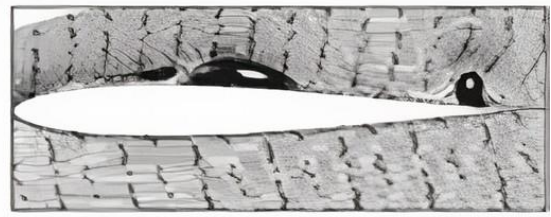

(h) a $6.0^{\circ}$

Fig. 1. Influence of angle of attack on laminar separation bubble (LSB) [4] 
Noises generated can be categorized into mechanical and aeroacoustic noise [9]. Mechanical noise can be mitigated by various ways such as isolating mechanical vibration and employing soundabsorbing materials [10]. However, aeroacoustic noises are more dominant and difficult to alleviate [11]. Aeroacoustic noise is divided into airfoil self-noise and turbulent inflow noise [9]. Airfoil selfnoise is further classified into Turbulent Boundary Layer - Trailing-Edge Noise, Laminar Boundary Layer - Vortex-Shedding Noise, Separation-Stall Noise, Trailing Edge Bluntness Vortex- Shedding Noise, and Tip Vortex Formation Noise (Figure 2) [12]. These noises are linked to subsonic conditions. For instance, at relatively low Re, instabilities that grow in the LBL interact with the trailing edge to generate noise. The interaction of turbulence layer with the trailing edge produces noise at high Re. For larger than zero angle of attack, the flow separates from the surface thus, radiating acoustic waves due to stall and reversal flow. Lastly, noise is produced by the vortex generated at the trailing edge as well as near the tip of the wing. The dominant source of the noise for various applications is Turbulent Boundary Layer - Trailing-Edge Noise [12,13], despite its contributions were initially neglected [13]. Turbulent Boundary Layer -Trailing-Edge Noise is caused by scattering of turbulence fluctuations within the boundary layer at the trailing edge thus, resulting in the radiation of the noise [9]. The sound pressure intensity of the trailing edge noise is related to varies flow characteristics so as to understand the noise phenomena better. The equation and the concept are well explained by the author [12].
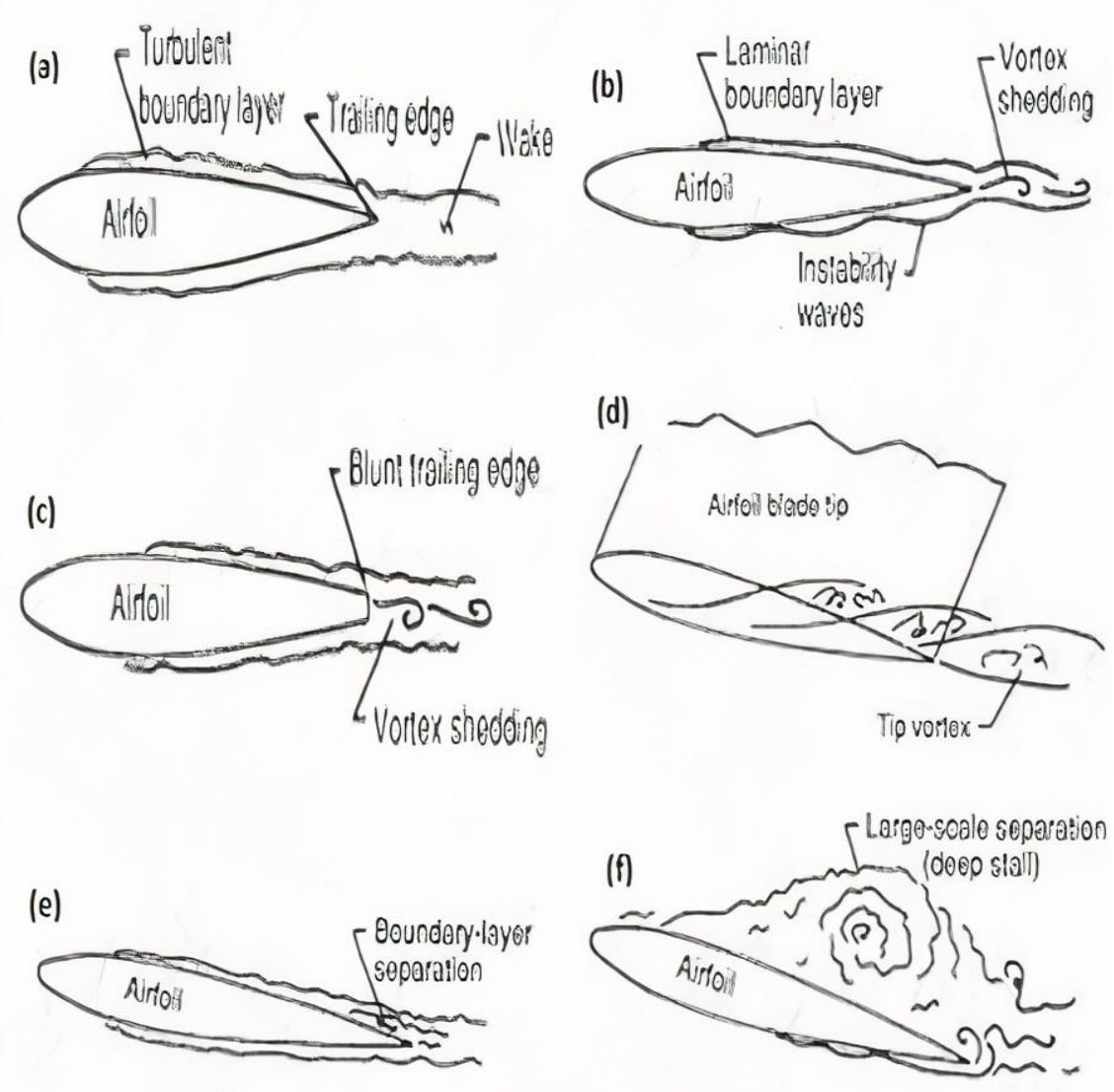

Fig. 2. Types of airfoil self-noise [9] 
Airfoils operating at low to moderate Reynolds numbers generate whistle-like tonal noise [2]. Whistle noise may be found on blunt bodies or airfoil-shaped structures such as buildings, fans, turbines, and aircraft wings. The discrete tonal noise emitting from a sharp trailing edge was initially identified from isolated airfoils operating at moderate Reynolds numbers [14-16]. For low to moderate Reynolds number, where micro-wind turbines, small UAV and compressor as well as cooling fans operate; airfoil produces tonal and broadband noises in the vicinity of the trailing edge. Broadband noise is either caused by the interaction of boundary layer turbulence and trailing edge, inflow turbulence and leading-edge interaction, and vortex-related conditions [17]. The existence of discrete tones and multiple tones was found in the spectrum [18-20]. Noise spectra generated show a primary tone $\left(f_{n}, \max \right)$ and a series of secondary tones $\left(f_{n}\right)$ as well as broadband hump $\left(f_{s}\right)$ defined as the centre frequency of the broadband noise component [21], as shown by Figure 3. In another study, airfoil tonal noise is composed of broadband noise with centered frequency and a superposition of discrete tonal noise [22]. Noticeable tonal noise is produced whenever peak frequency is at least $40 \mathrm{~dB}$ [2]. High-intensity tonal noise is generally more annoying as compared to broadband noise. An airfoil generates whistle-like noise by complying with a few criteria (Figure 4)

i. Laminar separation bubble amplifies TS-waves immensely near the trailing edge.

ii. Tonal noise is more effective whenever laminar separation bubble remains closer to the trailing edge of an airfoil.

iii. Flow reattachment near the trailing edge must not be inhibited by strong adverse pressure gradient.

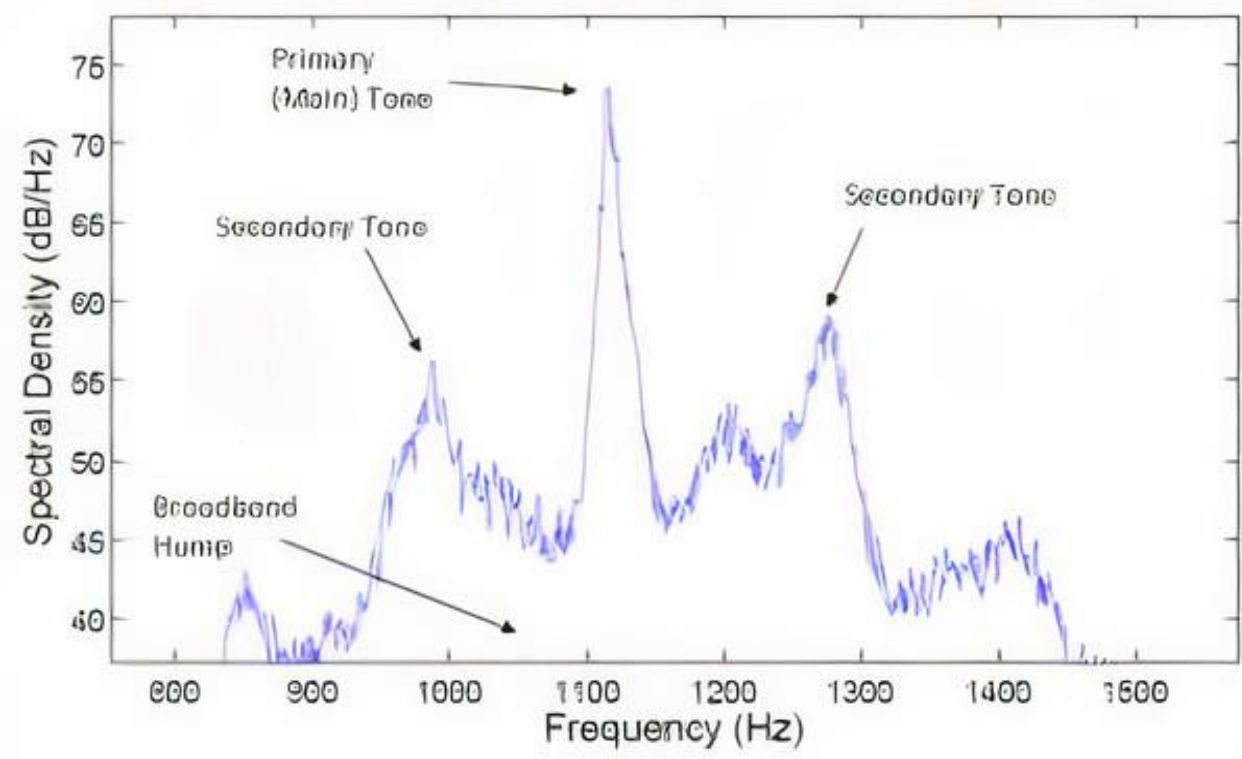

Fig. 3. Noise spectra generated on the airfoil surface [21] 


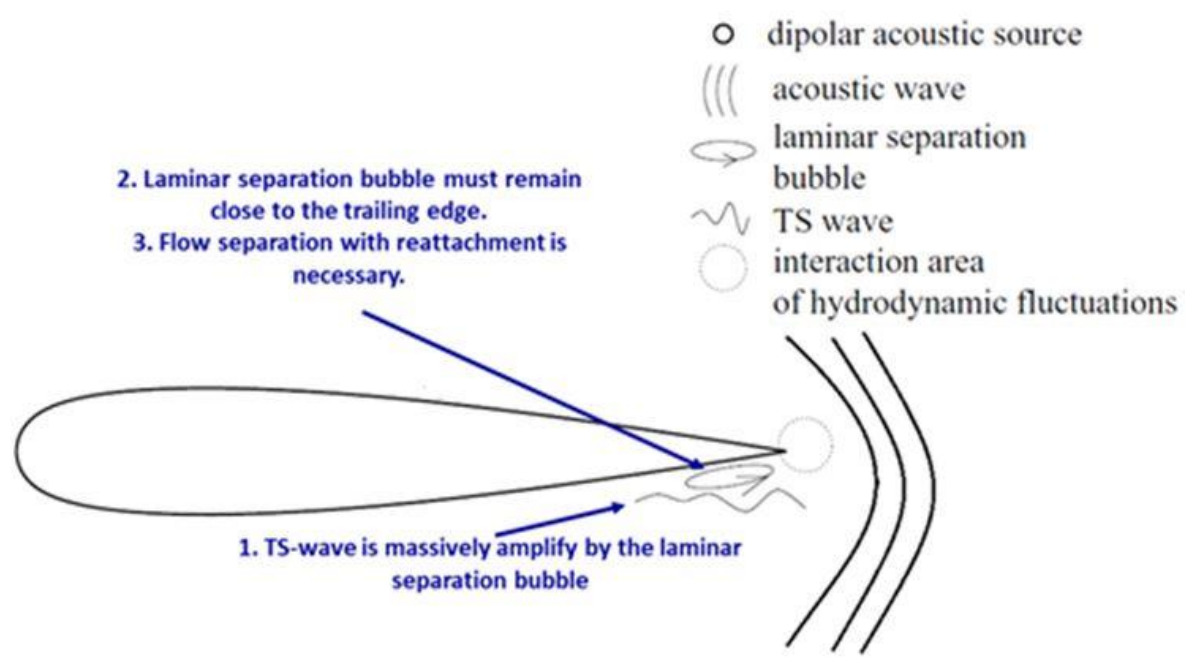

Fig. 4. Distinct whistle-like tonal noise generated from the airfoil [23]

Several researchers focused on detail understanding of the noise generation of an airfoil. The first empirical model to predict average frequency of the discrete tone is presented by Eq. (1) [20]. This was based on vortex shedding noise. The scaling law presented was modeled after measurement were obtained from NACA0012 and NACA0018.

$f=0.011 U^{1.5} / \sqrt{c U}$

where $\mathrm{U}$ is the freestream velocity, $\mathrm{c}$ is the chord length and $\mathrm{v}$ is kinematic viscosity of the fluid. On the other hand, discrete frequency as shown in Eq. (2) is presented considering the tone group $n$ to describe the phase condition. This was proposed because vortex shedding is not enough to explain the noise phenomena. A feedback mechanism was suggested to explain the phase condition instead [24].

$f=6.85 n U^{0.8}$

In addition, it was observed that noise is composed of broadband noise around the centered frequency $\left(f_{s}\right)$ and discrete components $\left(f_{n}\right)$ which are equidistant from each other. Experimentally, the centered frequency of the broadband noise $\left(f_{s}\right)$ on NACA0012 at a range of freestream velocities at $\alpha=0^{\circ}$ is as shown in Eq. (3) [19]. Displacement thickness at the trailing edge and freestream velocity is denoted as $\delta_{T E}$ and $U$ respectively. Strouhal number $\left(s t_{S}\right)$ was determined as 0.048 .

$f_{S}=s t_{S} U / \delta_{T E}$

Following another experimental study on NACA0012, a model to calculate the spectral peak was investigated at angles of attack ranging from $0^{\circ}$ to $25.2^{\circ}$, as shown by Eq. (4) [12].

$f_{S}=s t^{\prime} U / \delta_{P}$

Effective angle of attack as well as the Reynolds number is associated with Strouhal number st' and boundary layer height on the pressure side $\delta_{P}$. The empirical model can predict well the primary noise from a wall-mounted finite airfoil [25]. 
Past research shows that laminar separation bubble affects airfoil aerodynamic performance and contributes to airfoil tonal noise. Its formation, size, and location are affected by both angles of attack and Reynolds number. It is necessary to conduct a flow visualization experiment to study the physical flow characteristic to investigate the airfoil tonal noise mechanism. Existing empirical models have limitations in predicting airfoil-self noise generated at different angles of attack at low Reynolds number [2]. Flow measurement and visualization of the flow structure around the airfoil is necessary. Without these, the generation of the airfoil whistle noise may not be understood and may inhibit an in-depth explanation of the measured results. Alternatively, analysis of dynamic cases such as oscillations of the trailing edge has gained interest recently [25]. It has also been proven that it has significant effects on the noise generated $[26,27]$.

\section{Noise Generation Mechanism}

\subsection{Noise Generation}

Distinct airfoil tonal noise is said to be generated by the amplification of naturally developed laminar boundary layer instability by the laminar separation bubble near the airfoil trailing edge. However, this condition may not be entirely necessary for airfoil tonal emission. The airfoil tonal noise begins with significantly small instabilities known as Tollmien-Schlichting waves (TS-waves) that naturally occur inside the laminar boundary layer on the airfoil pressure side. The TS-waves instabilities undergo massive amplification by the laminar separation bubble near the trailing edge [28]. Consequently, noise is generated as the amplified instabilities pass the airfoil trailing edge. This phenomenon is commonly found at low-to-moderate Reynolds numbers [2]. Yet in another study, flow structure, laminar boundary layer initially but unsteady disturbances (T-S Wave) appear that initiate transition to turbulence, then boundary layer may also separate depending on flow condition causing the oscillation of the shear layer. Finally, the unsteady flows on both sides of the airfoil interact at the trailing edge forming a complex wake [29].

Airfoil noises are due to vortex shedding located at a short distance downstream from the trailing edge [20]. Similarly, vortex shedding due to laminar boundary layer instabilities and blunt trailing edges are the dominant sources of airfoil self-noise [11]. Moreover, the interaction of wake and boundary layers was also associated with the airfoil tonal noise [30]. The discrete tones at the trailing edge are linked to the laminar boundary layer located on the pressure surface [31].

Acoustic disturbances (T-S Waves) originating at the sharp trailing edge propagate downstream along the airfoil wake. Upon reaching sufficient magnitude, they induce lateral oscillations in the wake resulting in the emission of acoustic waves. A fraction of the acoustic wave energy is propagated upstream to the pressure side of the airfoil near the trailing edge, forcing the boundary layer to oscillate, thereby completing a feedback loop [24]. Likewise, acoustic waves travel upstream to complete a hydrodynamic and acoustic feedback loop. The end location of the feedback loop is the point at which the boundary layer instabilities on the airfoil profile originate [32]. However, another research proposes that hydrodynamic fluctuations propagate upstream to a point where the hydrodynamic instabilities are formed. This point is at which the flow velocity is the maximum in the laminar boundary layer. The hydrodynamic fluctuations will be amplified if the frequencies of the acoustic waves and hydrodynamic fluctuations are in phase. Hence, the hydrodynamics instabilities propagate downstream to complete the feedback loop [19]. As the unstable boundary layer forms, T-S waves continue growing and propagating toward the trailing edge before rolling up into a vortex. Thus, a scattered source oscillating at the same frequency as the T-S wave extends upstream to approximately half the chord at which the boundary layer becomes unstable, resulting in the narrowband acoustic tones. These shows that the feedback mechanism is based on a vortex shedding 
process [33]. Periodic variations of the velocity field in the separating region, followed by upwash and downwash cause the periodic formation of a vortex. Tonal noise is due to an adverse pressure gradient sufficiently small to allow instability waves to grow slowly thus, propagating upstream toward the point of boundary layer instability, initiating a feedback loop [34]. A secondary feedback loop exists when the laminar boundary layer separates. The T-S Waves interact with trailing edge forming a dipolar acoustic source. The waves then travel upstream forming a feedback loop. No tonal noise is generated with a sufficient turbulent boundary layer [35].

\subsection{Factors Affecting Airfoil Noise.}

A slight increase in velocity will increase the primary tone frequency by $U^{0.8}$ for symmetrical NACA airfoil operating at a moderate Reynolds number. Moreover, the primary tone instantly jumps to a higher frequency at certain velocities, forming a 0.8 power relationship thus, forming a ladder-like structure. $U^{0.8}$ curves vary for different Reynolds number and angles of attack. The frequency-tone relationship forms $U^{1.5}$ curve. Therefore, overall frequency of primary tone can be written as Eq. (1) [20].

Varying the Reynolds number also affects the primary tone amplitude and quantity. The primary tone decreases if the Reynolds number is increased at zero angles of attack [19]. At low Reynolds number, high intensity centered frequency $\left(f_{s}\right)$ is accompanied by more pronounced secondary discrete tonal noise of frequency $\left(f_{n}\right)$ as freestream velocity increases [2]. Whereas at moderate Reynolds number, multiple tones superimposed on a broadband hump are generated [30]. Yet another suggestion was higher Mach number increases the amplitude and the frequency of the tone.

The flow structure is asymmetric for a non-zero angle of attack. Thus, the boundary layer grows at different rates on both the suction and pressure sides. The suction side initially separate due to the unsteady behavior of the flow compared to the pressure side, which remains laminar over for relatively small angle of attack [30]. Airfoil tonal noise gradually decreases as the angle of attack increases from $\alpha=0^{0}$ before disappearing beyond $\alpha=5^{0}$ [2]. This might be explained due to the late transition on the pressure side and early separation on the suction side. Hence, causing the flow to be dominated by turbulent flow.

The reverse flow region is not displayed by thinner NACA 0012 airfoils [29]. This shows the influence of the airfoil thickness on the flow structure. Similarly, increasing airfoil thickness would increase low-frequency noise on the suction and pressure sides [36]. In addition, camber increases low-frequency noise on the suction side and decreases the noise on the pressure side [36]. Thicker blunt trailing edges would have a lower tonal frequency compared to thinner ones at the same velocity [37]. Thicker airfoils produce higher amplitude, whereas thinner ones emit higher frequency tones [30]. Experimentally, noise increases as the bevel angle are increased until $8^{\circ}$. However, the noise reduces gradually at higher angles. Beyond $20^{\circ}$, the noise drops below the baseline of $0^{\circ}$ [38].

\section{Flow and Noise Control}

\subsection{Flow Control}

Laminar Flow Control (LFC) is one method that forces a certain amount of energy into the flow to match the flow disturbance [41]. Due to unstable nature of the laminar flow, re-energizing it would enhance its stability on the surface of the model.

Natural Laminar Flow (NLF) is an alternative way of reducing drag [40]. This method employs a favorable pressure gradient to delay transition [40] by shaping the flow contour [41]. This is meant to reduce the momentum-thickness Reynolds number hence altering the transition point. It is 
passively applied compared to active LFC. It is strenuous to employ NLF in real applications due to the excessive pressure drag associated with it [41].

NFL becomes ineffective, especially when considering a 3D model. Since boundary instability is known as crossflow vortex, this causes the boundary-layer state near the wing leading edge to be turbulence. Moreover, it is indicated that the modern NFL system can overcome this issue by decreasing the leading-edge radius. Thus, decreasing the chordwise extent of the crossflow region and therefore providing a more rapid acceleration of the flow over the wing. The author has also suggested that NLF suffers from insect contamination and ice adherence to the system when applied to actual models [40].

Hybrid Laminar Flow Control (HLFC) is a significant advancement made in the development of LFC technology. LFC is a complex method, which involves suction (ducts, flutes, and pump source) over the whole-wing chord. HLFC integrates the concepts of NLF with LFC to reduce suction requirements and reduce system complexity [40].

\subsection{Influence on Aerodynamic Performance}

One of the usual methods used and researched regarding LFC is suction and blowing. However, unblown holes usually introduce roughness on the surface. Skin roughness might also be caused by several factors such as contaminations, manufacturing constraints, and coatings.

LFC provides skin friction reduction of about $75 \%$ compared to the solid plate; moreover, this reduces total skin reduction to about 1-2\%, specifically on the engine nacelles [41]. This reduction translates to less engine power requirement hence reduction in emission as well as the noise generated.

Another novel concept of porosity is micro-blowing technology (MBT). He has considered the unblown porous material to be MBT, if the ratio of its skin friction compared to the non-porous one is less than 1.1, indicating $10 \%$ increase in the drag compared to the solid model [42]. This shows that a porous surface always has more drag compared to the solid model. Even $8 \%$ porous which is considered aerodynamically smooth has a drag coefficient greater than non-porous plate [43]. Three parameters have been analyzed independent of each other to show the effect of each parameter individually. The parameters are hole diameter, aspect ratio, and porosity of the material. Based on the evaluation, the lowest skin friction ratio is acquired at Reynolds number based on the hole diameter of about 400, an aspect ratio of roughly 6, and porosity percentage within 13 to 23 [42]. Increasing the plenum depth would increase the drag coefficient. Moreover, small and closely parked holes would also increase drag coefficient because of the interactions and vortices in the holes [43].

Furthermore, it has been stated that blowing reduces aerodynamic efficiency while suction increases the aerodynamic efficiency on the suction side of the airfoil [44]. The authors also affirmed that blowing improves the aerodynamic efficiency on the pressure side of the airfoil. Similarly, passive blowing destabilizes the boundary layer while suction stabilizes it [45].

Roughness noise is generated due to the surface roughness. One of the parameters used to describe surface roughness is roughness height and roughness density. Roughness height and roughness density have been proven to affect far-field noise with roughness height having more effect [45]. On the other hand, an increase in the noise $[45,46]$ and overall sound pressure level $[45]$ at high frequency is associated with surface roughness noise, which is more significant at this range of frequency compared to the solid model. Moreover, roughness noise exceeds the trailing edge noise at sufficiently high frequency [45]. Surface roughness strengthens the acoustic sources at relatively high frequencies. Thus, the energy in the boundary layer may be scattered to radiate sound 
into the far-field [45]. Therefore, skin roughness should be considered in the analysis and design of the airfoil noise.

\subsection{Noise Control}

Some researchers worked on the porous models and presented the effect of the noise generated by the model. The terms used to define the porous material varies from one author to another. For example, porosity, permeability, resistivity, porosity constant, non-dimensionless permeability, flow control severity, and tortuosity. Porosity is the measure of the void space in a material. Permeability describes the ability of a material to transmit fluid through the void spaces. Resistivity indicates the ability of a material to oppose the flow through its void spaces. Noise generated at the trailing edge is mostly associated with the speed of sound, the density of the fluid, dynamic viscosity, chord length, span, angle of attack, airfoil profile, free-stream velocity, porosity, observer's distance from the surface, frequency, flow resistivity, boundary layer thickness and tortuosity [47]. From these properties, 11 dimensionless quantities were defined, which are normalized mean square sound pressure, chord-based Reynolds number, chord-based Strouhal number, Mach number, acoustical Rayleigh number, porosity, tortuosity, length ratios, and angle of attack [47]. In addition, materials can be described completely based on airflow resistivity, volume porosity, tortuosity, thermal permeability, characteristic viscous dimension, and thermal characteristic dimension [48]. However, the author attested that airflow resistivity and volume porosity influence the noise around the airfoil the most.

Pores facilitate the formation of crossflow and vortex shedding; the magnitude depends on the features related to the hole parameters. Eventually, this will affect the turbulence structures [49]. The noise generated can be minimized if a smaller pore size with sub-millimeter diameter, medium to large porosity, and/or small porous coverage is utilized. With those parameters, the flow oozes through the porous channel and surface. Hence, interfering with the boundary layer and lowers the radiated noise near the trailing edge [50]. The dominant noise source is close to the trailing edge. Therefore, smaller-sized pores can suppress vortex shedding noise and turbulent noise near the trailing edges [50]. Moreover, high-frequency noise near the trailing edges can significantly reduce with sub-millimetre pores [50]. In addition, porous materials generate noise when air flows over the transition of solid and porous parts. This source increases with the increased permeability [49]. Another reason for the additional noise is surface roughness, low porosity, and vibration of the trailing edge [51]. On top of all that, many porous models such as metal foams produced less noise compared to the baseline [51].

The thickness of the porous skin must be greater than the diameter of the holes; this gives an aspect ratio greater than one [52]. Moreover, they also reasoned that the hole diameter must be less than the boundary layer thickness [51,52], sharp and broadened noise is produced whenever the depth is at least twice the average boundary layer thickness [50]. The noise generated over the airfoil is directly proportional to the volume of the turbulence through the trailing edge [53]. Consequently, this will enhance the efficiency of the porous portion. In addition, sharp tones are dominant if the ratio of distance connecting the holes and the pore diameter is less than or equal to 1.5 . On the contrary, broadened tones are dominant whenever the ratio is more than 1.5 [50]. Airfoil trailing edge is categorized as sharp when the ratio of hole diameter to the boundary layer displacement thickness is less than 0.3 , and blunt trailing edge when it is more than 0.3 [54].

Moderate permeability provides the highest noise reduction [50,55]. Higher permeability has negligible resistance, thus regarded as a shortened chord. Whereas lower permeability behaves like a solid surface, thus giving almost the same results as the solid model. 
Moreover, noise reduction is observed by increasing the material's resistivity within low to medium frequencies; the same observation was made at high frequency for solid material $[55,56]$. On the other hand, an increase in the noise at high frequency is associated with surface roughness noise, which is more significant than the solid model [57].

For a partially porous airfoil study, increasing resistivity while reducing the extent of the porous region would increase lift coefficient and decrease drag coefficient [51,58]. Increasing the extent of the porous region from the trailing edge towards the leading edge will increase both boundary layer thickness and turbulence intensity, resulting in more wake deficit after the trailing edge [59]. Also, increasing extent of the porous region will reduce the lift coefficient over the airfoil surface [60]. A study suggested that a small extend of the porous region showed a reduction in noise over the airfoil [61] of a considerable 3.7\% coverage [50]. Slightly increasing the extent of the porous coverage will reduce noise radiation. However, sub-millimetre pores have more effect on the reduction of noise [50]. Additionally, material selection is an important criterion to achieve significant noise reduction [51]. It was also mentioned that a porous surface reduces the peak pressure fluctuation resulting in the reduction of noise intensity by weakening the strength of the Strouhal-shedding frequency. Moreover, correct placement of the porous region on the airfoil's surface can suppress the vortex roll-up, which will reduce pressure fluctuation and peak swirl velocity; consequently, reducing the generated noise [62]. Furthermore, smooth porosity distribution eliminates sudden changes in the acoustic impedance, which is known to be the dominant cause of the trailing edge noise [9].

Increasing flow control severity would increase the viscous region while decreasing the logarithmic region. The logarithmic region disappears when flow control severity is greater than 6 [63]. The highest noise reduction of about $5 \mathrm{~dB}$ is achieved at a flow control severity of 6 and the suction angle of $70^{\circ}$.

At considerably low Reynolds number $\left(5 \times 10^{3}\right)$, blowing reduces far-field noise above the trailing edge, and the reduction rate depends on the blowing velocity [30]. On the other hand, suction increases the far-field noise, and this trend is independent of the free stream velocity. Moreover, blowing at the trailing edge reduces the pressure fluctuation, suppressing the acoustic scattering near the trailing edge region [30]. This reduces airfoil noise directly since acoustic scattering is one of the sources of airfoil noise.

In addition, at relatively low Reynolds number (105) and zero angles of attack, suction reduces the spectrum related to secondary tones but increases the primary tonal peak [30]. They also claimed that blowing increases both the primary and secondary tones with only a slight increase in the former. Moreover, a slight increase in speed or at a constant Reynolds number, suction, and blowing reduces the frequencies and amplitude of the primary peak. However, the disturbance created by suction and blowing causes higher secondary peaks, especially suction amplifies the broadband noise. Furthermore, increasing the angle of attack from zero to three showed that blowing increases the primary tonal peaks and reduces the amplitudes of the secondary tones. In contrast, suction reduces the amplitude of the primary peak and increases the secondary tones.

Since the laminar flow is maintained using this technique along the transition and turbulence region of the flow, the boundary layer is measured using different methods such as hot-wire measurement immediately after suction and blower are applied. This technique will aid in visualizing the trend and behavior of the flow after applying blowing or suction. 


\section{Parametric Designs}

\subsection{Design Based on Skin Friction}

Three parameters were analyzed independently of each other to show the effect of each parameter individually. The parameters are hole diameter, aspect ratio, and porosity of the material. Aspect ratio is the ratio of the skin thickness to the hole diameter. Porosity is defined as the measure of the void space relative to the area covered by the apertures. Another term used in this study was Reynolds number based on the hole diameter. This study involves the diameter of the hole as the characteristic length exposed to the flow.

Based on the evaluation, the lowest skin friction ratio acquired at Reynolds number based on the hole diameter of about 400 shows the skin friction increases drastically above this value, whereas a slight increase in skin friction below this value. The aspect ratio of 6 gave the lowest skin friction ratio, where the skin friction ratio increases below and above this value. Porosity within $13 \%$ to $23 \%$ provides a minimum value of skin friction ratio, whereas an increase in skin friction ratio is observed for porosity above $25 \%$. Figures 5, 6, and 7 illustrate the above statements.

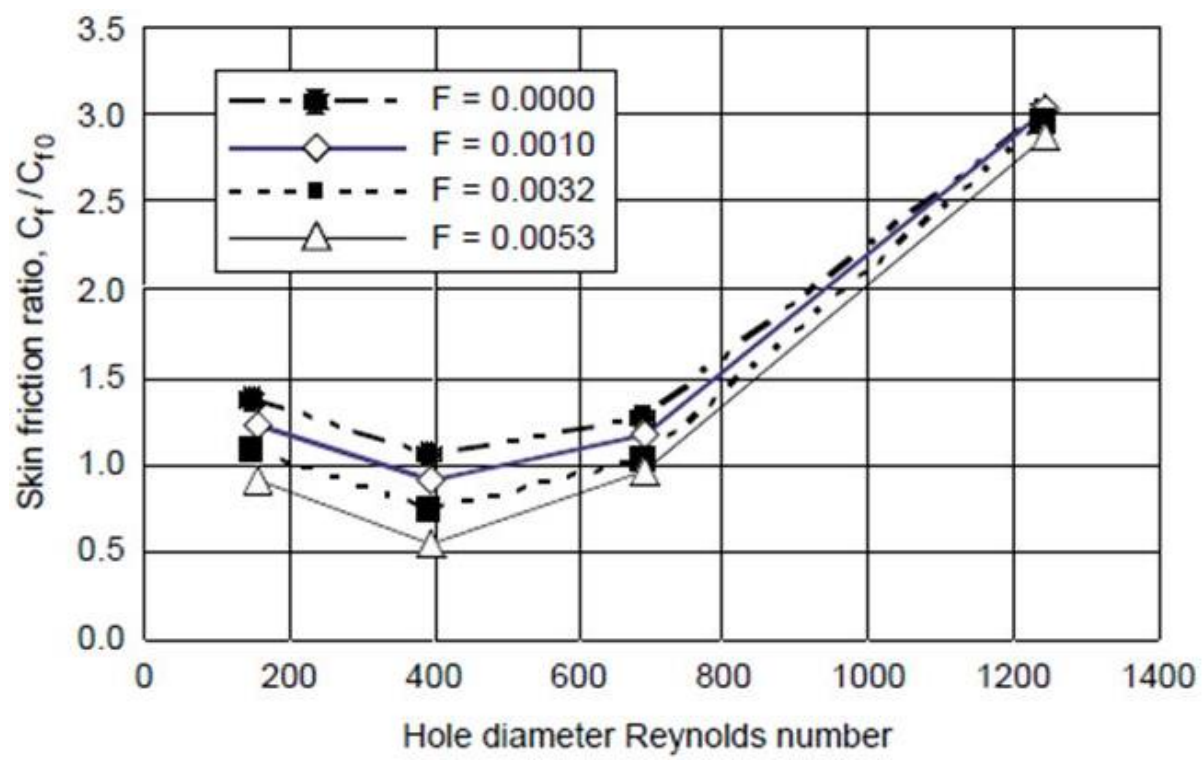

Fig. 5. Skin friction ratio with respect to hole diameter Reynolds number [42]

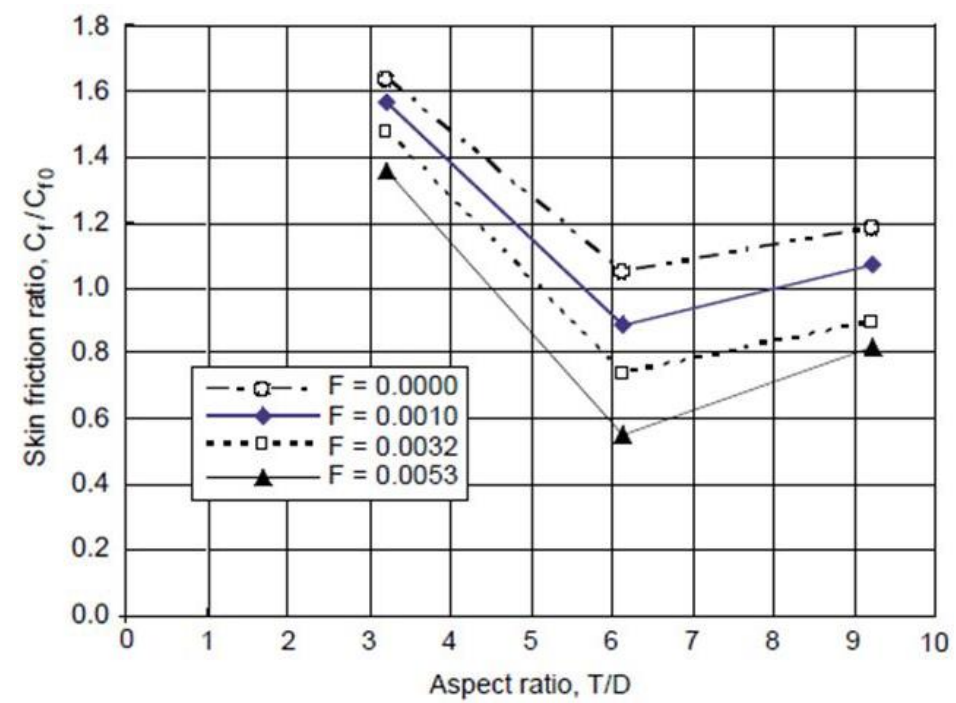

Fig. 6. Skin friction ratio with respect to aspect ratio [42] 
The three parameters, hole sizes, and the distribution can be designed to reduce the effect of skin friction. Additionally, skin roughness contributes to the noise at high frequency [57]. However, it is difficult to fulfill all conditions during the designing process because of many factors such as manufacturing constraints, restrictions based on the objectives of the study, etc. Therefore, we can optimize the parameters based on the aims of the study and the constraints. Finally, the parameters can be the prime component in the design of porous dimensions and arrangement.

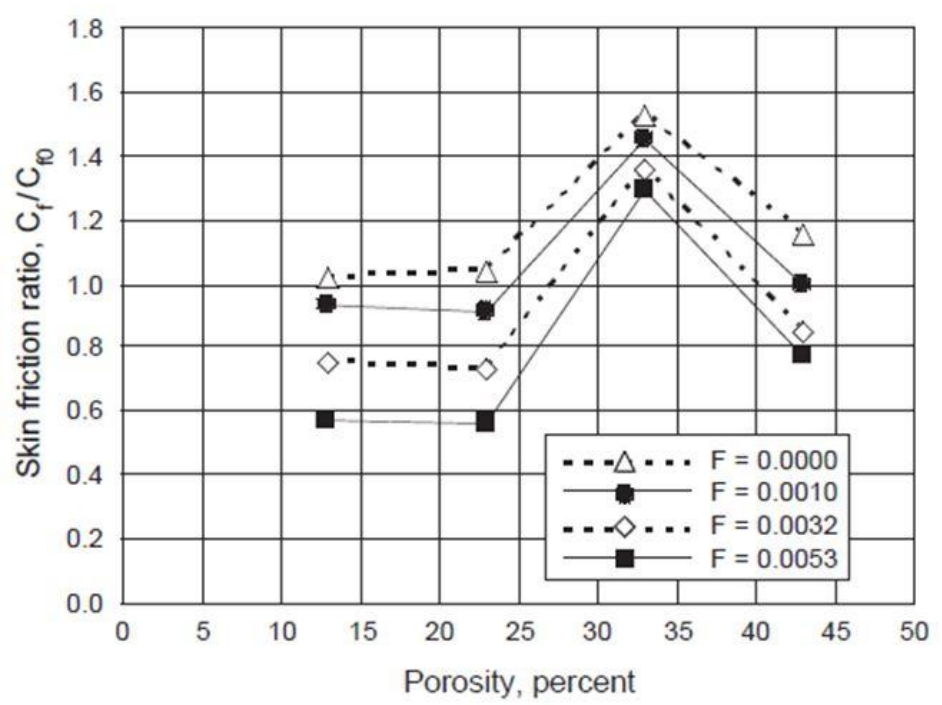

Fig. 7. Skin friction ratio with respect to porosity [42]

\subsection{Design Based on Aperture Parameters}

Based on Howe's theory [64], several assumptions were made. Among others, no consideration on the viscous effects, flow independent on the porosity, low Mach number, and the size of the eddies larger than the hole diameter, non-dimensional aperture parameter is related to the radiated trailing edge noise as follows

$\lambda=8 / \pi^{2} \varepsilon l / R$

where $\lambda$ is the non-dimensional aperture parameter, $\varepsilon$ is the porosity evaluated based on aperture area over the total porous area, $I$ is the extend of the porous area measured from the trailing edge, and $\mathrm{R}$ is the radius of the aperture.

Howe focused on two cases whereby one has uniform aperture distribution throughout its porous surface while the aperture of the second case increases linearly towards the trailing edge. Based on his analysis, he has recommended 0.89 and 1.25 as the optimum values of the non-dimensional aperture parameter for case 1 and case 2 respectively. These values provide significant noise reduction with a series of peaks in noise reduction at high frequency for case 1, whereas uniform noise reduction is observed for case 2 . However, experiments must be performed carefully when boundary layer turbulence is affected; the boundary layer might generate different noise sources. 


\subsection{Design Based on Flow Characteristics}

An equation at the boundary condition for the generation of a disturbance by suction and blower through a hole is defined in Eq. (6) [40].

$V=f(x) \exp (-i \omega t)$

where $\omega$ the frequency of the disturbance, $f(x)$ is the shape of the suction and blowing distribution, and $V$ is the resulting wall-normal velocity component at the wall.

The suction and blowing are regulated via suction and blower speed, and the size and inclination of the hole. It is laborious to solve some of the applications at hand. However, the problem can be solved by relating the normal velocity and aperture shape if it is separated into simple algebraic equations.

\section{Concluding Remarks}

The study of self-noise is bio-inspired, especially by owl wings and feathers, which have unique properties responsible for its silent flight. For instance, several features such as fringes and downy wing surfaces have shown significant noise reduction within audible range of their prey and themselves. However, detail physical analysis is needed for better understanding. Nonetheless, appropriate analysis is needed since the owl combines various features and behaviors during flight. The study may interconnect the puzzle of silent flight in the future.

Overall, noise on the airfoil surface is linked to vortex shedding, instabilities on the surface, and a feedback mechanism. In addition, acoustic scattering is minimized by reducing the extent of the porous region from the trailing edge while increasing resistivity. Moreover, blowing might also be another means of reducing noise near the trailing edges. Some of the parameters that affect the noise generated are freestream velocity, Reynolds number, angle of attack, and the airfoil profile.

The causes and the most optimum techniques to mitigate the airfoil noises are not well covered despite extensive research. Moreover, the flow topology needs to be understood clearly to explain the noise characteristics and interpret the performance rationally. In other words, the sources and noise mechanism may be explained and elaborated well when the features are presented accordingly. Subsequently, analysis of dynamic cases such as oscillations of the trailing edge has gained interest recently. It has also been proven that it has significant effects on the noise generated.

\section{Acknowledgement}

The research was supported by the Ministry of Education (MOE) through Fundamental Research Grant Scheme (FRGS/1/2018/TK09/UIAM/03/4). We would also like to thank financial support by IIUM under the KOE Postgraduate Tuition Fee Waiver Scheme 2019 (TFW2019).

\section{References}

[1] Jaworski, Justin W., and Nigel Peake. "Aeroacoustics of silent owl flight." Annual Review of Fluid Mechanics 52 (2020): 395-420. https://doi.org/10.1146/annurev-fluid-010518-040436

[2] Andan, Amelda Dianne, and Duck-Joo Lee. "Discrete Tonal Noise of NACA0015 Airfoil at Low Reynolds Number." Journal of Advanced Research in Fluid Mechanics and Thermal Sciences 53, no. 1 (2019): 129-145.

[3] Crivellini, Andrea, Valerio D'Alessandro, Daniele Di Benedetto, Sergio Montelpare, and Renato Ricci. "Study of laminar separation bubble on low Reynolds number operating airfoils: RANS modelling by means of an highaccuracy solver and experimental verification." In Journal of Physics: Conference Series, vol. 501, no. 1, p. 012024. IOP Publishing, 2014. https://doi.org/10.1088/1742-6596/501/1/012024 
[4] Lei, Juanmian, Feng Guo, and Can Huang. "Numerical study of separation on the trailing edge of a symmetrical airfoil at a low Reynolds number." Chinese Journal of Aeronautics 26, no. 4 (2013): 918-925. https://doi.org/10.1016/j.cja.2013.06.005

[5] Kurelek, John. "Transition in a Laminar Separation Bubble and the Effect of Acoustic Excitation." Master's thesis, University of Waterloo, 2016.

[6] Kim, Dong-Ha, and Jo-Won Chang. "Low-Reynolds-number effect on the aerodynamic characteristics of a pitching NACA0012 airfoil." Aerospace Science and Technology 32, no. 1 (2014): 162-168. https://doi.org/10.1016/i.ast.2013.08.018

[7] Plogmann, B., A. Herrig, and W. Würz. "Experimental investigations of a trailing edge noise feedback mechanism on a NACA 0012 airfoil." Experiments in fluids 54, no. 5 (2013): 1-14. https://doi.org/10.1007/s00348-013-1480-z

[8] Ma, Dongli, Yanping Zhao, Yuhang Qiao, and Guanxiong Li. "Effects of relative thickness on aerodynamic characteristics of airfoil at a low Reynolds number." Chinese Journal of Aeronautics 28, no. 4 (2015): 1003-1015. https://doi.org/10.1016/i.cja.2015.05.012

[9] Barone, Matthew F. "Survey of techniques for reduction of wind turbine blade trailing edge noise." Prepared for Sandia National Laboratory (2011). https://doi.org/10.2172/1029824

[10] Crivellini, Andrea, Valerio D'Alessandro, Daniele Di Benedetto, Sergio Montelpare, and Renato Ricci. "Study of laminar separation bubble on low Reynolds number operating airfoils: RANS modelling by means of an highaccuracy solver and experimental verification." In Journal of Physics: Conference Series, vol. 501, no. 1, p. 012024. IOP Publishing, 2014. https://doi.org/10.1088/1742-6596/501/1/012024

[11] Jakobsen, J. Andersen. "Aerodynamical noise from wind turbine generators. Experiments with modification of full scale rotors." (1993).

[12] Brooks, Thomas F., D. Stuart Pope, and Michael A. Marcolini. Airfoil self-noise and prediction. Vol. 1218. Washington, DC: National Aeronautics and Space Administration, Office of Management, Scientific and Technical Information Division, 1989.

[13] Guo, Yueping, and Russell H. Thomas. "On aircraft trailing edge noise." In 25th AIAA/CEAS Aeroacoustics Conference, p. 2610. 2019. https://doi.org/10.2514/6.2019-2610

[14] Smith, D. L., R. P. Paxson, R. D. Talmadge, and E. R. Hotz. Measurements of the radiated noise from sailplanes. AIR FORCE FLIGHT DYNAMICS LAB WRIGHT-PATTERSON AFB OH VEHICLE DYNAMICS DIV, 1970. https://doi.org/10.21236/AD0709689

[15] Clark, Larry Trumbull. "The radiation of sound from an airfoil immersed in a laminar flow." (1971): 366-376. https://doi.org/10.1115/1.3445595

[16] Hersh, Alan S., and Richard E. Hayden. "Aerodynamic sound radiation from lifting surfaces with and without leadingedge serrations." (1971).

[17] Casalino, D., E. Grande, G. Romani, D. Ragni, and F. Avallone. "Towards the definition of a benchmark for low Reynolds number propeller aeroacoustics." In Journal of Physics: Conference Series, vol. 1909, no. 1, p. 012013. IOP Publishing, 2021. https://doi.org/10.1088/1742-6596/1909/1/012013

[18] Williams, JE Ffowcs, and L. H. Hall. "Aerodynamic sound generation by turbulent flow in the vicinity of a scattering half plane." Journal of fluid mechanics 40, no. 4 (1970): 657-670. https://doi.org/10.1017/S0022112070000368

[19] Arbey, Henri, and J. Bataille. "Noise generated by airfoil profiles placed in a uniform laminar flow." Journal of Fluid Mechanics 134 (1983): 33-47. https://doi.org/10.1017/S0022112083003201

[20] Paterson, Robert W., Paul G. Vogt, Martin R. Fink, and C. Lee Munch. "Vortex noise of isolated airfoils." Journal of Aircraft 10, no. 5 (1973): 296-302. https://doi.org/10.2514/3.60229

[21] Arcondoulis, E., C. Doolan, and Anthony C. Zander. "Airfoil noise measurements at various angles of attack and low Reynolds number." Proceeding of ACOUSTICS (2009): 23-25.

[22] Schumacher, Karn L., Con J. Doolan, and Richard M. Kelso. "The effect of acoustic forcing on an airfoil tonal noise mechanism." The Journal of the Acoustical Society of America 136, no. 2 (2014): EL78-EL83. https://doi.org/10.1121/1.4885485

[23] Pröbsting, S., J. Serpieri, and F. Scarano. "Experimental investigation of aerofoil tonal noise generation." Journal of Fluid Mechanics 747 (2014): 656. https://doi.org/10.1017/ifm.2014.156

[24] Tam, Christopher KW. "Discrete tones of isolated airfoils." The Journal of the Acoustical Society of America 55, no. 6 (1974): 1173-1177. https://doi.org/10.1121/1.1914682

[25] Moreau, Danielle J., Con J. Doolan, W. Nathan Alexander, Timothy W. Meyers, and William J. Devenport. "Wallmounted finite airfoil-noise production and prediction." AIAA Journal 54, no. 5 (2016): 1637-1651. https://doi.org/10.2514/1.J054493

[26] Zhou, Teng, Yuhao Sun, Ryu Fattah, Xin Zhang, and Xun Huang. "An experimental study of trailing edge noise from a pitching airfoil." The Journal of the Acoustical Society of America 145, no. 4 (2019): 2009-2021. https://doi.org/10.1121/1.5094898 
[27] Manela, A. "On the acoustic radiation of a pitching airfoil." Physics of Fluids 25, no. 7 (2013): 071906. https://doi.org/10.1063/1.4816295

[28] Park, Donghun, and Seung O. Park. "Study of tonal noise behavior of an airfoil by using parabolized stability equations." Theoretical and Computational Fluid Dynamics 27, no. 1 (2013): 71-88. https://doi.org/10.1007/s00162-011-0254-6

[29] Sandberg, R. D., L. E. Jones, N. D. Sandham, and P. F. Joseph. "Direct numerical simulations of tonal noise generated by laminar flow past airfoils." Journal of Sound and Vibration 320, no. 4-5 (2009): 838-858. https://doi.org/10.1016/i.jsv.2008.09.003

[30] Ramirez, Walter A., and William Wolf. "The effects of suction and blowing on tonal noise generation by blunt trailing edges." In 21st AIAA/CEAS Aeroacoustics Conference, p. 2364. 2015. https://doi.org/10.2514/6.2015-2364

[31] Fink, Martin R. "Prediction of airfoil tone frequencies." Journal of Aircraft 12, no. 2 (1975): 118-120. https://doi.org/10.2514/3.44421

[32] Fink, Martin R., Robert H. Schlinker, and Roy K. Amiet. "Prediction of rotating-blade vortex noise from noise of nonrotating blades." Final Report United Technologies Research Center (1976).

[33] Lowson, Martin, Alan McAlpine, and Emma Nash. "The generation of boundary layer instability noise on aerofoils." In 36th AIAA Aerospace Sciences Meeting and Exhibit, p. 627. 1998. https://doi.org/10.2514/6.1998-627

[34] Nakano, T., N. Fujisawa, and S. Lee. "Measurement of tonal-noise characteristics and periodic flow structure around NACA0018 airfoil." Experiments in Fluids 40, no. 3 (2006): 482-490. https://doi.org/10.1007/s00348-005-0089-2

[35] Desquesnes, G., M. Terracol, and P. Sagaut. "Numerical investigation of the tone noise mechanism over laminar airfoils." Journal of Fluid Mechanics 591 (2007): 155-182. https://doi.org/10.1017/S0022112007007896

[36] Lee, Seongkyu. "The effect of airfoil shape on trailing edge noise." Journal of Theoretical and Computational Acoustics 27, no. 02 (2019): 1850020. https://doi.org/10.1142/S2591728518500202

[37] Tam, Christopher, and Hongbin Ju. "Airfoil tones at moderate reynolds number: a computational study." In 17th AIAA/CEAS Aeroacoustics Conference (32nd AIAA Aeroacoustics Conference), p. 2711.2011. https://doi.org/10.2514/6.2011-2711

[38] Celik, Alper, J. Luke Bowen, and Mahdi Azarpeyvand. "Effect of trailing-edge bevel on the aeroacoustics of a flatplate." Physics of Fluids 32, no. 10 (2020): 105116. https://doi.org/10.1063/5.0024248

[39] KERSCHEN, EDWARD. "Boundary layer receptivity." In 12th Aeroacoustic Conference, p. 1109.1989. https://doi.org/10.2514/6.1989-1109

[40] Joslin, Ronald D. "Overview of laminar flow control." (1998). https://doi.org/10.1146/annurev.fluid.30.1.1

[41] Tillman, T. G. "Drag reduction on a large-scale nacelle using micro-porous blowing." UTRC Report R97-4.910 1 (1997).

[42] Hwang, Danny. "Review of research into the concept of the microblowing technique for turbulent skin friction reduction." Progress in Aerospace Sciences 40, no. $8 \quad$ (2004): https://doi.org/10.1016/i.paerosci.2005.01.002

[43] Wilkinson, S. "Influence of wall permeability on turbulent boundary-layer properties." In 21st Aerospace Sciences Meeting, p. 294. 1983. https://doi.org/10.2514/6.1983-294

[44] Atzori, M., R. Vinuesa, P. Schlatter, D. Gatti, A. Stroh, and B. Frohnapfel. "Effects of uniform blowing and suction on turbulent wing boundary layers." In Proceedings of the European Drag Reduction and Flow Control Meeting (EDRFCM). 2019.

[45] Liu, Yu, Ann Dowling, and Ho-Chul Shin. "Effects of surface roughness on airframe noise." In 12th AIAA/CEAS Aeroacoustics Conference (27th AIAA Aeroacoustics Conference), p. 2510. 2006. https://doi.org/10.2514/6.2006$\underline{2510}$

[46] Geyer, Thomas, Ennes Sarradj, and Christoph Fritzsche. "Porous airfoils: noise reduction and boundary layer effects." International journal of aeroacoustics 9, no. 6 (2010): 787-820. https://doi.org/10.1260/1475$\underline{472 X .9 .6 .787}$

[47] Sarradj, Ennes, and Thomas F. Geyer. "Airfoil noise analysis using symbolic regression." In 19th AIAA/CEAS Aeroacoustics Conference, p. 2012. 2013. https://doi.org/10.2514/6.2013-2012

[48] Geyer, Thomas. "Trailing edge noise generation of porous airfoils." (2011).

[49] Bernicke, Paul, R. A. D. Akkermans, Varun B. Ananthan, Roland Ewert, Jürgen Dierke, and L. Rossian. "A zonal noise prediction method for trailing-edge noise with a porous model." International Journal of Heat and Fluid Flow 80 (2019): 108469. https://doi.org/10.1016/j.ijheatfluidflow.2019.108469

[50] Zhang, Minghui, and Tze Pei Chong. "Experimental investigation of the impact of porous parameters on trailingedge noise." Journal of Sound and Vibration 489 (2020): 115694. https://doi.org/10.1016/j.jsv.2020.115694

[51] Geyer, Thomas Fritz, and Ennes Sarradj. "Self noise reduction and aerodynamics of airfoils with porous trailing edges." In Acoustics, vol. 1, no. 2, pp. 393-409. Multidisciplinary Digital Publishing Institute, 2019. https://doi.org/10.3390/acoustics1020022 
[52] Frink, Neal T., Daryl L. Bonhaus, Veer N. Vatsa, Steven XS Bauer, and Ana F. Tinetti. "Boundary condition for simulation of flow over porous surfaces." Journal of Aircraft 40, no. 4 (2003): 692-698. https://doi.org/10.2514/2.3147

[53] Lilley, Geoffrey. "A study of the silent flight of the owl." In 4th AIAA/CEAS aeroacoustics conference, p. 2340.1998. https://doi.org/10.2514/6.1998-2340

[54] Herr, Michaela. "Design criteria for low-noise trailing-edges." In 13th AIAA/CEAS Aeroacoustics Conference (28th AIAA Aeroacoustics Conference), p. 3470. 2007. https://doi.org/10.2514/6.2007-3470

[55] Bae, Youngmin, and Young J. Moon. "Effect of passive porous surface on the trailing-edge noise." Physics of Fluids 23, no. 12 (2011): 126101. https://doi.org/10.1063/1.3662447

[56] Geyer, Thomas, Ennes Sarradj, and Christoph Fritzsche. "Measurement of the noise generation at the trailing edge of porous airfoils." Experiments in fluids 48, no. 2 (2010): 291-308. https://doi.org/10.1007/s00348-009-0739-x

[57] Geyer, Thomas, Ennes Sarradj, and Christoph Fritzsche. "Porous airfoils: noise reduction and boundary layer effects." International journal of aeroacoustics 9, no. 6 (2010): 787-820. https://doi.org/10.1260/1475$\underline{472 X .9 .6 .787}$

[58] Geyer, Thomas, and Ennes Sarradj. "Noise reduction and aerodynamics of airfoils with porous trailing edges." In INTER-NOISE and NOISE-CON Congress and Conference Proceedings, vol. 258, no. 7, pp. 641-650. Institute of Noise Control Engineering, 2018.

[59] Geyer, Thomas F., and Ennes Sarradj. "Trailing edge noise of partially porous airfoils." In 20th AIAA/CEAS aeroacoustics conference, p. 3039. 2014. https://doi.org/10.2514/6.2014-3039

[60] Aldheeb, Mohammed, Waqar Asrar, Erwin Sulaeman, and Ashraf A. Omar. "Aerodynamics of porous airfoils and wings." Acta Mechanica 229, no. 9 (2018): 3915-3933. https://doi.org/10.1007/s00707-018-2203-6

[61] Khorrami, Mehdi R., and Meelan M. Choudhari (2003). Application of passive porous treatment to slat trailing edge noise. NASA Technical Memorandum 212416.

[62] Revell, James, James Revell, Herbert Kuntz, Frank Balena, Clifton Horne, Bruce Storms, Robert Dougherty et al. "Trailing-edge flap noise reduction by porous acoustic treatment." In 3rd AIAA/CEAS aeroacoustics conference, p. 1646. 1997. https://doi.org/10.2514/6.1997-1646

[63] Szoke, Máté, Daniele Fiscaletti, and Mahdi Azarpeyvand. "Uniform Suction for the Reduction of the Trailing-Edge Noise." In 25th AIAA/CEAS Aeroacoustics Conference, p. 2651. 2019. https://doi.org/10.2514/6.2019-2651

[64] Howe, M. S. "On the added mass of a perforated shell, with application to the generation of aerodynamic sound by a perforated trailing edge." Proceedings of the Royal Society of London. A. Mathematical and Physical Sciences 365, no. 1721 (1979): 209-233. https://doi.org/10.1098/rspa.1979.0014 